\title{
Absolute continuity of the spectrum of the periodic Schrödinger operator in a layer and in a smooth cylinder
}

\author{
N. Filonov \\ I. Kachkovskiy*
}

\begin{abstract}
We consider the Schrödinger operator $H=-\Delta+V$ in a layer or in a $d$-dimensional cylinder. The potential $V$ is assumed to be periodic with respect to some lattice. We establish the absolute continuity of $H$, assuming $V \in L_{p \text { loc }}$, where $p$ is a real number greater than $d / 2$ in the case of a layer, and $p>\max (d / 2, d-2)$ for the cylinder. ${ }^{1}$
\end{abstract}

\section{Introduction}

Let $M$ be a smooth $k$-dimensional compact Riemannian manifold, let also

$$
\Xi=M \times \mathbb{R}^{m}, \quad d:=\operatorname{dim} \Xi=k+m .
$$

We are interested in the type of the spectrum of the Schrödinger operator $H=-\Delta+V$ in a cylinder $\Xi$. The function $V$ is supposed to be periodic. If $M$ is a manifold with boundary, we study the operator $H$ with various boundary conditions at $\partial \Xi=\partial M \times \mathbb{R}^{m}$. We are going to prove that, under some assumptions on $V$, the spectrum of $H$ is absolutely continuous (see Theorems 2.1 and 2.2 below).

The points of $\Xi$ are denoted by $(x, y), x \in M, y \in \mathbb{R}^{m}$. Let $\Gamma$ be a lattice in $\mathbb{R}^{m}$,

$$
\Gamma=\left\{l=\sum_{j=1}^{m} l_{j} b_{j}, \quad l_{j} \in \mathbb{Z}\right\},
$$

where $\left\{b_{j}\right\}_{j=1}^{m}$ is a basis of $\mathbb{R}^{m}$. Assume that $V$ is periodic over the "longitudinal" variables:

$$
V(x, y+l)=V(x, y), \quad x \in M, y \in \mathbb{R}^{m}, l \in \Gamma .
$$

Thanks to $V$ being periodic, it is enough to know $V$ on $M \times \Omega$, where

$$
\Omega=\left\{y=\sum_{j=1}^{m} y_{j} b_{j}, \quad y_{j} \in[0,1)\right\}
$$

is an elementary cell of $\Gamma$.

*The first author was supported by RFBR grant 08-01-00209.

${ }^{1}$ Keywords: Schrödinger operator, Periodic Coefficients, Absolutely Continuous Spectrum. 
Let us introduce the reader to the main results regarding absolute continuity of $H$. Usually, in the sufficient conditions it is assumed that the potential $V$ belongs to $L_{p}(M \times \Omega)$ or to a Lorentz space $L_{p, \infty}^{0}(M \times \Omega)$. We recall that if $N$ is a set of finite measure, then $L_{p}(N) \subset$ $L_{p, \infty}^{0}(N) \subset L_{p-\varepsilon}(N)$ for all $\varepsilon>0$.

The two-dimensional case, $d=2$ ( $\Xi$ is a whole plane or a strip), has been studied in much detail. In $[1,10,7]$, the absolute continuity of $H$ is proved for $V \in L_{p}, p>1$. From now on, we consider only $d \geqslant 3$.

The case of $k=0$, corresponding to the operator in the whole space, is also well studied. In [8], the absolute continuity is established in the "critical" case $V \in L_{d / 2, \infty}^{0}(\Omega)$ for all $d \geqslant 3$ (see also [3]). In [13], the case $k=1$ ( $M$ is a line segment, $\Xi$ is a plane-parallel layer) is studied, and for $V \in L_{p, \infty}^{0}(M \times \Omega)$, where $p=\max (d / 2, d-2)$, the absolute continuity of $H$ is obtained. The author also considers the third type boundary condition. Finally, the case $k \geqslant 2$ is studied in [4], and it is established that $H$ is absolutely continuous if $V \in L_{d-1}(M \times \Omega)$.

In the present paper, we prove (see Theorem 2.1 below) the absolute continuity of $H$ with $V \in L_{p}(M \times \Omega)$ for all $p>d / 2$ in the following cases: 1) $\left.\partial M=\emptyset ; 2\right) M$ is a line segment, $k=1$; 3) $d=3$ or 4 . If $M$ is a manifold with boundary, $k>1$, and $d>4$, we obtain only $V \in L_{d-2}(M \times \Omega)$ as a sufficient condition. In the case $k=1$ we also consider the third type boundary condition (see Theorem 2.2).

All mentioned results are obtained using the Thomas scheme [14], its key point is to study the operator family

$$
H(\xi)=-\Delta_{x}+\left(-i \nabla_{y}+\xi\right)^{*}\left(-i \nabla_{y}+\xi\right)+V(x, y)
$$

where $\xi$ is called quasimomentum. To obtain the resolvent estimates for the free operator $H_{0}(\xi)$, corresponding to $V=0$, we use the spectral cluster estimates from [12] (the idea of using these estimates arose in [8]).

\section{Formulation of the result}

Let $M$ be a compact smooth Riemannian manifold with or without boundary, $\operatorname{dim} M=k$. Consider a $d$-dimensional cylinder

$$
\Xi=M \times \mathbb{R}^{m}, \quad d=k+m \geqslant 3 .
$$

Let $\Gamma$ be a lattice (1.1), let $\Omega$ be a cell (1.3), and let $V(x, y)$ be a real-valued function, satisfying (1.2). Assume that

$$
V \in L_{d / 2}(M \times \Omega) .
$$

Consider the following quadratic form in $L_{2}(\Xi)$ :

$$
h[u, u]=\int_{\Xi}\left(|\nabla u(x, y)|^{2}+V(x, y)|u(x, y)|^{2}\right) d x d y, \quad \operatorname{Dom} h=H^{1}(\Xi) .
$$

If $M$ has a boundary, $\partial M \neq \emptyset$, then we denote $(2.2)$ by $h_{N}$. In this case we are also going to study a form $h_{D}=\left.h_{N}\right|_{H_{0}^{1}(\Xi)}$. 
It is well known that, assuming (2.1), the form $h$ (resp. $h_{N}, h_{D}$ ) is closed and semi-bounded from below. In $L_{2}(\Xi)$, it corresponds to a semi-bounded operator $H$ (resp. $H_{N}, H_{D}$ ), which is called the Schrödinger operator in $\Xi$ (resp. the Schrödinger operator with Dirichlet or Neumann boundary conditions).

Theorem 2.1. Let $M$ be a compact smooth Riemannian manifold with or without boundary, $\operatorname{dim} M=k, \Xi=M \times \mathbb{R}^{m}, d=k+m \geqslant 3$. Let $\Gamma$ be a lattice (1.1), let $V$ be a real-valued $\Gamma$-periodic function in $\Xi$. Assume that $V \in L_{p}(M \times \Omega)$, where

- $p>d / 2$, if $\partial M=\emptyset$;

- $p>d / 2$, if $\partial M \neq \emptyset$ and $k=1$ ( $M$ is a line segment);

- $p>d / 2$, if $\partial M \neq \emptyset$ and $d=3$ or $d=4$;

- $p>d-2$, if $\partial M \neq \emptyset$ and $d \geqslant 5$.

Then the spectra of $H(\partial M=\emptyset), H_{N}$ and $H_{D}(\partial M \neq \emptyset)$ are absolutely continuous.

In the case of a layer ( $M$ is a line segment), Suslina's result [13] (see Theorem 4.5 below) allows us to consider the case of the third type boundary condition. Let $k=1, \Xi=[0, a] \times \mathbb{R}^{m}$, let also $\sigma$ be a real $\Gamma$-periodic function on $\partial \Xi=\{0 ; a\} \times \mathbb{R}^{m}$. Consider a quadratic form

$$
\begin{array}{r}
h_{\sigma}[u, u]=\int_{\Xi}\left(|\nabla u(x, y)|^{2}+V(x, y)|u(x, y)|^{2}\right) d x d y \\
+\int_{\mathbb{R}^{m}}\left(\sigma(a, y)|u(a, y)|^{2}-\sigma(0, y)|u(0, y)|^{2}\right) d y, \quad \operatorname{Dom} h_{\sigma}=H^{1}(\Xi) .
\end{array}
$$

If $\sigma \in L_{m}(\{0, a\} \times \Omega)$, then the form (2.3) is closed and semi-bounded from below (see [9]). In the case $\sigma=0$ the form $h_{\sigma}$ coincides with $h_{N}$.

Theorem 2.2. Let $\Xi=[0, a] \times \mathbb{R}^{m}, d=m+1 \geqslant 3$, let $\Gamma$ be a lattice (1.1). Let $V$ be $a$ $\Gamma$-periodic function on $\Xi, V \in L_{p}([0, a] \times \Omega)$ with $p>d / 2$. Let $\sigma$ be a $\Gamma$-periodic function on $\partial \Xi$, satisfying

$$
\sigma \in L_{q}(\{0, a\} \times \Omega), \quad \text { where } q=2 \text { for } d=3, \quad q=2 d-2 \text { for } d \geqslant 4 .
$$

Then the spectrum of the Schrödinger operator $H_{\sigma}$, corresponding to the form (2.3), is absolutely continuous.

Remark 2.3. Theorem 2.1 can be reformulated in the matrix case. Let $V$ be an $(n \times n)$ matrix-valued function on $\Xi$ such that $V(x, y)^{*}=V(x, y),(1.2)$ holds, and $V \in L_{p}(M \times \Omega)$, $p>d / 2$. The quadratic form

$$
h[u, u]=\int_{\Xi}\left(|\nabla u(x, y)|^{2}+\langle V(x, y) u(x, y), u(x, y)\rangle\right) d x d y
$$

is closed and semi-bounded on the domains $H^{1}\left(\Xi, \mathbb{C}^{n}\right)$ and $H_{0}^{1}\left(\Xi, \mathbb{C}^{n}\right)$. These forms correspond to the self-adjoint operators $H, H_{N}, H_{D}$ in $L_{2}\left(\Xi, \mathbb{C}^{n}\right)$. In the cases of a manifold without 
boundary, a layer, and 3- and 4-dimensional cylinders, the spectra of such operators are absolutely continuous. In the case of a $d$-dimensional cylinder, $d>4$, the spectra of $H_{N}$ and $H_{D}$ are absolutely continuous whenever $V \in L_{p}(M \times \Omega), p>d-2$. The proof of Theorem 2.1 is valid for the matrix case without changes. A matrix analog of Theorem 2.2 can also be obtained.

It is convenient for us to interpret $\Omega$ as an $m$-dimensional torus $\mathbb{T}=\mathbb{R}^{m} / \Gamma$. Let us introduce an additional parameter $\xi \in \mathbb{C}^{m}$, and consider the following quadratic forms. In the case of a manifold without boundary let

$$
h(\xi)[v, v]=\int_{M \times \Omega}\left(\left|\nabla_{x} v\right|^{2}+\left\langle\left(\nabla_{y}+i \xi\right) v,\left(\nabla_{y}+i \bar{\xi}\right) v\right\rangle+V|v|^{2}\right) d x d y
$$

$$
\operatorname{Dom} h(\xi)=H^{1}(M \times \mathbb{T}) .
$$

If $\partial M \neq \emptyset$, then the form $(2.5)$ will be denoted by $h_{N}(\xi)$, and let also

$$
h_{D}(\xi)=\left.h_{N}(\xi)\right|_{H_{0}^{1}(M \times \mathbb{T})} .
$$

In the case of a layer, $\Xi=[0, a] \times \mathbb{R}^{m}$, consider also a form

$$
\begin{array}{r}
h_{\sigma}(\xi)[v, v]=h_{N}(\xi)[v, v]+\int_{\Omega}\left(\sigma(a, y)|v(a, y)|^{2}-\sigma(0, y)|v(0, y)|^{2}\right) d y, \\
\operatorname{Dom} h_{\sigma}(\xi)=H^{1}([0, a] \times \mathbb{T}) .
\end{array}
$$

These forms are sectorial (the definition and main properties of sectoriality can be found in [5, Ch. VI, VII]), and they correspond to analytic operator families $H(\xi), H_{N}(\xi), H_{D}(\xi)$, and $H_{\sigma}(\xi)$ respectively. For real $\xi$, these operators are self-adjoint.

Let $b_{1}$ be the first vector in the basis of $\Gamma$. The conditions on the potential are dilatationinvariant, so we can assume $\left|b_{1}\right|=1$.

Theorem 2.4. Suppose the conditions of Theorem 2.1 or Theorem 2.2 are satisfied. Then, for every $\lambda \in \mathbb{C}$ and $\xi \in \mathbb{R}^{m}, \xi \perp b_{1}$, there exists $\tau_{0}$ such that for $|\tau|>\tau_{0}$ the operator $\left(H\left((\pi+i \tau) b_{1}+\xi\right)-\lambda I\right)$ is invertible and

$$
\left\|\left(H\left((\pi+i \tau) b_{1}+\xi\right)-\lambda I\right)^{-1}\right\| \leqslant C|\tau|^{-1} .
$$

We prove this Theorem in $§ 4$. In a standard way (see, for example, [2] or [6]) Theorem 2.4 implies Theorems 2.1 and 2.2 .

\section{Spectral cluster estimates}

For a self-adjoint operator $P$, we denote by $E_{k}(P)=E_{P}\left(\left[(k-1)^{2} ; k^{2}\right)\right)$ its spectral projector onto a subspace, corresponding to an interval $\left[(k-1)^{2} ; k^{2}\right)$. The following Theorem is proved in $[12]$.

Theorem 3.1. Let $N$ be a compact $C^{\infty}$-smooth d-dimensional Riemannian manifold without boundary, let $P$ be an elliptic second-order differential operator on $N$ with positive-definite symbol. Then

$$
\left\|E_{k}(P) f\right\|_{L_{2}(N)} \leqslant C k^{d(1 / p-1 / 2)-1 / 2}\|f\|_{L_{p}(N)}, \quad f \in L_{p}(N), \quad 1 \leqslant p \leqslant \frac{2(d+1)}{d+3} .
$$


By duality, this yields

Corollary 3.2. Under the assumptions of Theorem 3.1, the following inequality holds:

$$
\left\|E_{k}(P) f\right\|_{L_{q}(N)} \leqslant C k^{d(1 / 2-1 / q)-1 / 2}\|f\|_{L_{2}(N)}, \quad f \in L_{2}(N), \quad \frac{2(d+1)}{d-1} \leqslant q \leqslant+\infty .
$$

Theorem 3.3. Let $N_{0}$ be a compact smooth Riemannian manifold without boundary, $\operatorname{dim} N_{0}=$ $d-1$. Let $P_{0}$ be a second-order elliptic differential operator on $N_{0}$ with positive-definite symbol. Consider an elliptic operator $P=1 \otimes P_{0}-\frac{d^{2}}{d x^{2}} \otimes 1$ on a manifold $N=[0, a] \times N_{0}$ ( $x$ denotes a local coordinate on $[0, a])$. Then, for $P$ on $N$ with either Dirichlet or Neumann boundary conditions, the estimate (3.1) holds.

Proof. We shal give proof for the Dirichlet problem, the Neumann case is analogous. The statement of Theorem is invariant with respect to dilatations over $x$, so we can assume $a=\pi$. In this case, the spectral projector $E_{k}$ of $P$ is an integral operator with kernel

$$
K\left(x, x^{\prime}, y, y^{\prime}\right)=\sum_{j^{2}+\lambda_{n} \in\left[(k-1)^{2} ; k^{2}\right)} \frac{2}{\pi} \sin (j x) \sin \left(j x^{\prime}\right) \varphi_{n}(y) \bar{\varphi}_{n}\left(y^{\prime}\right),
$$

where $\left\{\lambda_{n}\right\},\left\{\varphi_{n}\right\}$ are eigenvalues and eigenfunctions of $P_{0}$. We introduce three operators: an operator $\widetilde{E}_{k}$, acting on functions from $L_{2}\left([0,2 \pi] \times N_{0}\right)$ as an integral operator with the same kernel (3.2), an operator of zero extension $T: L_{2}(N) \rightarrow L_{2}\left([0,2 \pi] \times N_{0}\right)$, and a restriction operator $S: L_{q}\left([0,2 \pi] \times N_{0}\right) \rightarrow L_{q}(N)$. Obviously, $E_{k}=S \widetilde{E}_{k} T$. Furthermore, $\widetilde{E}_{k}=\frac{1}{2 \pi}\left(\widetilde{E}_{k}^{(1)}-\right.$ $\left.\widetilde{E}_{k}^{(2)}\right)$, where $\widetilde{E}_{k}^{(1)}$ and $\widetilde{E}_{k}^{(2)}$ are integral operators with kernels

$$
\begin{aligned}
& K^{(1)}\left(x, x^{\prime}, y, y^{\prime}\right)=\sum_{j^{2}+\lambda_{n} \in\left[(k-1)^{2} ; k^{2}\right)}\left(e^{i j\left(x-x^{\prime}\right)}+e^{-i j\left(x-x^{\prime}\right)}\right) \varphi_{n}(y) \bar{\varphi}_{n}\left(y^{\prime}\right), \\
& K^{(2)}\left(x, x^{\prime}, y, y^{\prime}\right)=\sum_{j^{2}+\lambda_{n} \in\left[(k-1)^{2} ; k^{2}\right)}\left(e^{i j\left(x+x^{\prime}\right)}+e^{-i j\left(x+x^{\prime}\right)}\right) \varphi_{n}(y) \bar{\varphi}_{n}\left(y^{\prime}\right) .
\end{aligned}
$$

The operator $\widetilde{E}_{k}^{(1)}$ is a spectral projector of $-\frac{d^{2}}{d x^{2}} \otimes 1+1 \otimes P_{0}$ on $[0,2 \pi] \times N_{0}$ with periodic boundary conditions over $x$. The last operator is an elliptic operator on a manifold $S^{1} \times N_{0}$ without boundary, and it satisfies (3.1). Similarly, (3.1) holds for $\widetilde{E}_{k}^{(2)}$, and so for $\widetilde{E}_{k}$ and $E_{k}$.

The proof for the Neumann case can be obtained by replacing $\sin (j x)$ with $\cos (j x)$, in this case $\widetilde{E}_{k}=\frac{1}{2 \pi}\left(\widetilde{E}_{k}^{(1)}+\widetilde{E}_{k}^{(2)}\right)$.

In [11], the following result is proved.

Theorem 3.4. Let $N$ be a compact smooth Riemannian manifold with boundary, $\operatorname{dim} N=d \geqslant$ 3. Let $P$ be an elliptic second-order differential operator on $N$ with positive-definite symbol and with Dirichlet or Neumann boundary conditions. Then, for

$$
5 \leqslant q \leqslant \infty \text {, if } d=3 ; \quad 4 \leqslant q \leqslant \infty \text {, if } d \geqslant 4,
$$

the estimate (3.1) holds. For

$$
2 \leqslant q \leqslant 4, \quad d \geqslant 4
$$

the estimate is replaced with

$$
\left\|E_{k} f\right\|_{L_{q}(N)} \leqslant C k^{d(1 / 2-1 / q)+2 / q-1}\|f\|_{L_{2}(N)} .
$$




\section{Proof of Theorem 2.4}

For simplicity, denote $H\left((\pi+i \tau) b_{1}+\xi\right)$ by $H(\tau)$. Let

$$
H_{0}(\tau)=\left.H(\tau)\right|_{V=0, \sigma=0}, \quad H_{0}=H_{0}(0) .
$$

The operator $H_{0}$ is a self-adjoint second-order elliptic differential operator on a manifold $M \times \mathbb{T}$. Let $E_{k}$ denote its spectral projector onto $\left[(k-1)^{2} ; k^{2}\right)$. For a manifold $M$, we introduce

Condition $\mathrm{A}(q)$. M satisfies the property that for every $\xi \in \mathbb{R}^{m},\left\langle\xi, b_{1}\right\rangle=0$, there exist $\varepsilon>0$ and $C>0$ such that

$$
\left\|E_{k} f\right\|_{L_{q}(M \times \mathbb{T})} \leqslant C k^{1 / 2-\varepsilon}\|f\|_{L_{2}(M \times \mathbb{T})}, \quad \forall f \in L_{2}(M \times \mathbb{T}) .
$$

It is easy to see that $\mathrm{A}(q)$ implies $A(\tilde{q})$ if $\tilde{q}<q$.

Let $\left\{\mu_{j}\right\}$ and $\left\{\varphi_{j}(x)\right\}$ be eigenvalues and eigenfunctions of the Laplace operator $-\Delta_{x}$ on $M$ with the corresponding (Dirichlet or Neumann) boundary conditions. Then the eigenvalues of $H_{0}(\tau)$ are of the form

$$
h_{j, n}(\tau)=\left|n+\pi b_{1}+\xi\right|^{2}+\mu_{j}-\tau^{2}+2 i \tau\left\langle n+\pi b_{1}, b_{1}\right\rangle,
$$

and the normalized eigenfunctions are

$$
\varphi_{j, n}(x, y)=|\Omega|^{-1 / 2} \varphi_{j}(x) e^{i\langle n, y\rangle}, \quad j \in \mathbb{N}, n \in \widetilde{\Gamma},
$$

where $\widetilde{\Gamma}$ is the dual lattice,

$$
\tilde{\Gamma}=\left\{n=\sum_{j=1}^{m} n_{j} \tilde{b}_{j}, n_{j} \in \mathbb{Z}\right\}, \quad\left\langle b_{k}, \tilde{b}_{j}\right\rangle=2 \pi \delta_{k j} .
$$

Notice that $\left\langle n, b_{1}\right\rangle \in 2 \pi \mathbb{Z}$. This gives

$$
\left|h_{j, n}(\tau)\right| \geqslant\left|\operatorname{Im} h_{j, n}(\tau)\right|=2\left|\left\langle n+\pi b_{1}, b_{1}\right\rangle\right||\tau| \geqslant 2 \pi|\tau| .
$$

Then, for $|\tau|>0$, the operator $H_{0}(\tau)$ is invertible and

$$
\left\|H_{0}(\tau)^{-1}\right\| \leqslant(2 \pi|\tau|)^{-1}, \quad \tau \neq 0 .
$$

Consider also an operator $\left|H_{0}(\tau)\right|^{-1 / 2}$ such that

$$
\left|H_{0}(\tau)\right|^{-1 / 2} \varphi_{j, n}=\left|h_{j, n}(\tau)\right|^{-1 / 2} \varphi_{j, n} .
$$

The following Lemma is elementary.

Lemma 4.1. Let $0<\varepsilon<1 / 2$. Then the sums

$$
\sum_{k=1}^{\infty} \frac{k^{1-2 \varepsilon}}{\left|k^{2}-\tau^{2}\right|+|\tau|}, \quad \sum_{k=1}^{\infty} \frac{k^{1-2 \varepsilon}}{\left|(k-1)^{2}-\tau^{2}\right|+|\tau|}
$$

are finite and uniformly bounded with respect to $\tau$ for $|\tau|>1$. 
Proof. For certainty, consider the first sum. Without loss of generality, we can assume $\tau>0$. If $k^{2} \geqslant 2 \tau^{2}$, then the denominator can be replaced with $\frac{1}{2} k^{2}$, and this implies that the "tail" of the sum converges uniformly. Therefore, we may consider only $k^{2}<2 \tau^{2}$. In this case,

$$
\sum_{k<2 \tau} \frac{k^{1-2 \varepsilon}}{\left|k^{2}-\tau^{2}\right|+|\tau|} \leqslant 2|\tau|^{1-2 \varepsilon} \sum_{k<2 \tau} \frac{1}{\left|k^{2}-\tau^{2}\right|+|\tau|} \leqslant 2 \tau^{-2 \varepsilon} \sum_{k<2 \tau} \frac{1}{|k-\tau|+1} .
$$

The last sum is bounded, because

$$
\tau^{-2 \varepsilon} \int_{0}^{2 \tau} \frac{d k}{|k-\tau|+1}=2 \tau^{-2 \varepsilon} \int_{\tau}^{2 \tau} \frac{d k}{k-\tau+1}=2 \tau^{-2 \varepsilon} \ln (\tau+1) .
$$

Theorem 4.2. Assume that Condition $\mathrm{A}(q)$ holds. Then, for some $\tau_{0}>0$,

$$
\left\|\left|H_{0}(\tau)\right|^{-1 / 2} f\right\|_{L_{q}(M \times \mathbb{T})} \leqslant C\|f\|_{L_{2}(M \times \mathbb{T})}, \quad \forall|\tau|>\tau_{0}, f \in L_{2}(M \times \mathbb{T}) .
$$

Proof. Let $E_{k}$ be a spectral projector of $H_{0}$ onto $\left[(k-1)^{2} ; k^{2}\right)$. Then

$$
\begin{array}{r}
\left\|\left|H_{0}(\tau)\right|^{-1 / 2} f\right\|_{L_{q}(M \times \mathbb{T})} \leqslant \sum_{k=1}^{\infty}\left\|E_{k}\left|H_{0}(\tau)\right|^{-1 / 2} f\right\|_{L_{q}(M \times \mathbb{T})} \\
\leqslant C \sum_{k=1}^{\infty} k^{1 / 2-\varepsilon}\left\|E_{k}\left|H_{0}(\tau)\right|^{-1 / 2} f\right\|_{L_{2}(M \times \mathbb{T})} \leqslant C \sum_{k=1}^{\infty} k^{1 / 2-\varepsilon}\left\|E_{k}\left|H_{0}(\tau)\right|^{-1 / 2}\right\| \cdot\left\|E_{k} f\right\|_{L_{2}(M \times \mathbb{T})},
\end{array}
$$

from which, using Cauchy-Bunyakovsky-Schwarz inequality, we obtain

$$
\left\|\left|H_{0}(\tau)\right|^{-1 / 2} f\right\|_{L_{q}(M \times \mathbb{T})}^{2} \leqslant C\|f\|_{L_{2}(M \times \mathbb{T})}^{2} \sum_{k=1}^{\infty} k^{1-2 \varepsilon}\left\|E_{k}\left|H_{0}(\tau)\right|^{-1 / 2}\right\|^{2} .
$$

The eigenvalues of $H_{0}$ are $\left|n+\pi b_{1}+\xi\right|^{2}+\mu_{j}, n \in \widetilde{\Gamma}, j \in \mathbb{N}$. The range of $E_{k}$ corresponds to the pairs $(j, n)$ such that $(k-1)^{2} \leqslant\left|n+\pi b_{1}+\xi\right|^{2}+\mu_{j}<k^{2}$. So,

$$
\begin{aligned}
\left\|E_{k}\left|H_{0}(\tau)\right|^{-1 / 2}\right\|^{2}= & \max _{\left|n+\pi b_{1}+\xi\right|^{2}+\mu_{j} \in\left[(k-1)^{2} ; k^{2}\right)} \frac{1}{\left|h_{j, n}(\tau)\right|} \\
& \leqslant \max _{\left|n+\pi b_{1}+\xi\right|^{2}+\mu_{j} \in\left[(k-1)^{2} ; k^{2}\right)} \frac{\sqrt{2}}{\left|n+\pi b_{1}+\xi\right|^{2}+\mu_{j}-\tau^{2}|+| \tau \mid} .
\end{aligned}
$$

Finally, we need to show that the sum

$$
\sum_{k=1}^{\infty} \max _{\left|n+\pi b_{1}+\xi\right|^{2}+\mu_{j} \in\left[(k-1)^{2} ; k^{2}\right)} \frac{k^{1-2 \varepsilon}}{|| n+\pi b_{1}+\left.\xi\right|^{2}+\mu_{j}-\tau^{2}|+| \tau \mid}
$$

is finite and uniformly bounded for $|\tau|>\tau_{0}$.

To do this, we notice that in all the terms (maybe, all but one) we can replace $\left|n+\pi b_{1}+\xi\right|^{2}+$ $\mu_{j}$ with $(k-1)^{2}$ or $k^{2}$, and the term will not decrease, because, if $|\tau| \notin[k-1 ; k)$, then, after one of these substitutions, the denominator may only decrease. The term, for which $|\tau| \in[k-1 ; k)$, can be estimated by $C k^{-2 \varepsilon}$ and does not affect the convergence. So, it is enough to consider two sums (4.4): we replace $\left|n+\pi b_{1}+\xi\right|^{2}+\mu_{j}$ with $(k-1)^{2}$ in the first one, and with $k^{2}$ in the second one. Their boundness follows from Lemma 4.1. 
We need the following fact to prove Theorem 2.4:

Lemma 4.3. Let $(M, \mu)$ be a measurable space with $\sigma$-finite measure, let $V \in L_{p}(M), 1 \leqslant p<$ $\infty$. Then for every $\delta>0$ there exists $c(\delta)$ such that

$$
\int_{M}|V f g| d \mu \leqslant \delta\|f\|_{L_{2 p^{\prime}}(M)}\|g\|_{L_{2 p^{\prime}}(M)}+c(\delta)\|f\|_{L_{2}(M)}\|g\|_{L_{2}(M)}, \quad f, g \in L_{2 p^{\prime}}(M),
$$

where $p^{\prime}$ is the conjugate index to $p$.

Proof. The function $V$ can be expressed in the form

$$
V=V_{1}+V_{2}, \quad \text { where } \quad\left\|V_{1}\right\|_{L_{p}(M)} \leqslant \delta, \quad V_{2} \in L_{\infty}(M) .
$$

By Hölder inequality,

$$
\int_{M}|V f g| d \mu \leqslant \delta\|f\|_{L_{2 p^{\prime}}(M)}\|g\|_{L_{2 p^{\prime}}(M)}+\left\|V_{2}\right\|_{L_{\infty}(M)}\|f\|_{L_{2}(M)}\|g\|_{L_{2}(M)} .
$$

Theorem 4.4. Let $M$ satisfy $\mathrm{A}(q)$ for some $q \in(2,2 d /(d-2))$. Let $V \in L_{p}(M \times \mathbb{T})$, where $p=q /(q-2)$. Then the operator $(H(\tau)-\lambda I)$ is invertible for $|\tau|>\tau_{0}$, and $\left\|(H(\tau)-\lambda I)^{-1}\right\| \leqslant$ $C|\tau|^{-1}$.

Proof. The condition on $V$ is invariant with respect to adding a constant. So, without loss of generality, we can assume $\lambda=0$. It is enough to prove the following statement: for any $u \in \operatorname{Dom}(H(\tau)),\|u\|_{L_{2}(M \times \mathbb{T})}=1$, there exists $v \in \operatorname{Dom}(H(\tau)),\|v\|_{L_{2}(M \times \mathbb{T})}=1$, such that

$$
|(H(\tau) u, v)| \geqslant C|\tau|, \quad|\tau|>\tau_{0} .
$$

Let $H_{0}(\tau)=\Phi_{0}(\tau)\left|H_{0}(\tau)\right|$ be the polar decomposition of $H_{0}(\tau)$. We set

$$
v=\Phi_{0}(\tau) u \text {. }
$$

Then,

$$
\left(H_{0}(\tau) u, v\right)=\left(\left|H_{0}(\tau)\right| u, u\right) \geqslant 2 \pi|\tau|
$$

by (4.1), and

$$
\left(H_{0}(\tau) u, v\right)=\left\|\left|H_{0}(\tau)\right|^{1 / 2} u\right\|_{L_{2}(M \times \mathbb{T})}^{2}=\left\|\left|H_{0}(\tau)\right|^{1 / 2} v\right\|_{L_{2}(M \times \mathbb{T})}^{2} .
$$

Let us estimate the term $(V u, v)$ using Lemma 4.3 and Theorem 4.2:

$$
\begin{aligned}
|(V u, v)| & \leqslant \delta\|u\|_{L_{q}(M \times \mathbb{T})}\|v\|_{L_{q}(M \times \mathbb{T})}+c(\delta) \leqslant \\
& \leqslant C \delta\left\|\left.|| H_{0}(\tau)\right|^{1 / 2} v\right\|_{L_{2}(M \times \mathbb{T})}\left\|\left|H_{0}(\tau)\right|^{1 / 2} u\right\|_{L_{2}(M \times \mathbb{T})}+c(\delta)=C \delta\left(H_{0}(\tau) u, v\right)+c(\delta) .
\end{aligned}
$$

This implies

$|(H(\tau) u, v)| \geqslant(1-C \delta)\left(H_{0}(\tau) u, v\right)-c(\delta) \geqslant 2 \pi(1-C \delta)|\tau|-c(\delta) \geqslant C_{1}|\tau| \quad$ for $|\tau|>\tau_{0}, \delta<1 / C$. 
Proof of Theorem 2.4, the case of a manifold without boundary.

If $\partial M=\emptyset$, then Corollary 3.2 implies $\mathrm{A}(q)$ for all $q<2 d /(d-2)$. From Theorem 4.4, we get (2.6) for any $p>d / 2$.

Proof of Theorem 2.4, the case of Dirichlet or Neumann boundary conditions.

If $k=1$ ( $M$ is a line segment), then Theorem 3.3 again yields $\mathrm{A}(q)$ for all $q<2 d /(d-2)$. And all $p>d / 2$ are suitable.

If $d=3$, then Theorem 3.4 gives $\mathrm{A}(q)$ only if $q<6$, so we need $p>3 / 2$.

If $d \geqslant 4$, then, again by Theorem 3.4, Condition $\mathrm{A}(q)$ holds for $q<(2 d-4) /(d-3)$, and the corresponding condition on $V$ is $V \in L_{p}(M \times \Omega)$, where $p>d-2$.

To study the third type boundary condition, we use the following result from [13].

Theorem 4.5. Let $k=1, M=[0, a]$, and assume that $\sigma$ satisfies (2.4). Then

$$
\begin{array}{r}
\int_{\Omega}|\sigma(0, y)|\left|\left(\left|H_{0}(\tau)\right|^{-1 / 2} u\right)(0, y)\right|^{2} d y \\
+\int_{\Omega}|\sigma(a, y)|\left|\left(\left|H_{0}(\tau)\right|^{-1 / 2} u\right)(a, y)\right|^{2} d y \leqslant \tilde{c}(\tau)\|u\|_{L_{2}([0, a] \times \Omega)}^{2},
\end{array}
$$

where $\lim _{|\tau| \rightarrow \infty} \tilde{c}(\tau)=0$ uniformly over $\xi^{\prime}$ and $u \in L_{2}([0, a] \times \Omega)$.

Proof of Theorem 2.4, the case of the third type boundary condition.

Let $p>d / 2, q=2 p^{\prime}<2 d /(d-2)$. Theorem 3.3 guaranties $\mathrm{A}(q)$. Let $V \in L_{p}([0, a] \times \Omega)$.

For an arbitrary $u \in \operatorname{Dom}\left(H_{\sigma}\right),\|u\|_{L_{2}([0, a] \times \Omega)}=1$, let $v$ be defined by (4.5). Then

$$
\left(H_{\sigma}(\tau) u, v\right)=\left(H_{0}(\tau) u, v\right)+(V u, v)+\int_{\Omega} \sigma(a, y) u(a, y) \bar{v}(a, y) d y-\int_{\Omega} \sigma(0, y) u(0, y) \bar{v}(0, y) d y
$$

The first two terms are estimated in (4.7) and (4.6). Let us estimate the last one (the same can be done for the remaining term). Theorem 4.5 gives

$$
\begin{array}{r}
\left|\int_{\Omega} \sigma(0, y) u(0, y) \bar{v}(0, y) d y\right| \leqslant \frac{1}{2} \int_{\Omega}|\sigma(0, y)|\left(|u(0, y)|^{2}+|v(0, y)|^{2}\right) d y \\
\leqslant \frac{\tilde{c}(\tau)}{2}\left(\left\|\left|H_{0}(\tau)\right|^{1 / 2} u\right\|_{L_{2}([0, a] \times \Omega)}^{2}+\left\|\left|H_{0}(\tau)\right|^{1 / 2} v\right\|_{L_{2}([0, a] \times \Omega)}^{2}\right)=\tilde{c}(\tau)\left(H_{0}(\tau) u, v\right) .
\end{array}
$$

Hence,

$\left|\left(H_{\sigma}(\tau) u, v\right)\right| \geqslant\left(H_{0}(\tau) u, v\right)(1-C \delta-2 \tilde{c}(\tau))-c(\delta) \geqslant 2 \pi(1-C \delta-2 \tilde{c}(\tau))|\tau|-c(\delta), \quad|\tau|>\tau_{0}$

where $\delta$ and $\tau_{0}$ are chosen in such a way that $C \delta+2 \tilde{c}(\tau)<1,|\tau|>\tau_{0}$. The last estimate implies (2.6). 


\section{References}

[1] Birman M. Sh., Suslina T. A., Absolute continuity of a two-dimensional periodic magnetic Hamiltonian with discontinuous vector potential, Algebra i Analiz 10 (1998), no. 4, p. 1-36. English translation in St. Petersburg Math. J. 10 (1999), no. 4, p. 579-601.

[2] Birman M. Sh, Suslina T. A., Periodic magnetic Hamiltonian with variable metrics. Problem of absolute continuity, Algebra i Analiz, vol. 11 (1999), 2, pp. 1-40. English translation in St. Petersburg Math. J. 11 (2000), no. 2, p. 203-232.

[3] Danilov L. I., On absolute continuity of the spectrum of a periodic magnetic Schrödinger operator, J. Phys. A: Math. Theor. 42 (2009) 275204.

[4] N. Filonov, I. Kachkovskii, Absolute continuity of the spectrum of a periodic Schrödinger operator in a multidimensional cylinder, Algebra i Analiz, 21 (2009), no. 1, p. 133-152. English translation in St. Petersburg Math. J. 21 (2010), no. 1, p. 95-109.

[5] Kato T., Perturbation Theory for Linear Operators, Grundlehren der mathematischen Wissenschaften, Vol. 132. Berlin-Heidelberg-New York: Springer-Verlag, 1966.

[6] Reed M., Simon B., Methods of Modern Mathematical Physics, Vol 4: Analysis of Operators, Academic Press, New-York, 1978.

[7] Shargorodsky E., Sobolev A. V., Quasiconformal mappings and periodic spectral problems in dimension two, J. Anal. Math. 91 (2003), p. 67-103.

[8] Shen Z., On absolute continuity of the periodic Schrödinger operators, Intern. Math. Res. Notes (2001), no. 1, p. 1-31.

[9] Shterenberg R.G., Suslina T. A., Absolute continuity of the spectrum of the Schrödinger operator with the potential concentrated on a periodic system of hypersurfaces, Algebra i Analiz 13 (2001), no. 5, pp. 197-240. English translation in St. Petersburg Math. J. 13 (2002), no. 5, p. 859-891.

[10] Shterenberg R. G., Suslina T. A., Absolute continuity of the spectrum of the magnetic Schrödinger operator with a metric in a two-dimensional periodic waveguide. Algebra $\mathrm{i}$ Analiz 14 (2002), no. 2, pp. 159-206. English translation in St. Petersburg Math. J. 14 (2003), no. 2, p. 305-343.

[11] Smith H. F., Sogge C. D., On the $L_{p}$ norm of spectral clusters for compact manifolds with boundary, Acta Mathematica 198 (2007) no. 1, p. 107-153.

[12] Sogge C. D., Concerning the $L^{p}$ norm of spectral clusters for second-order elliptic operators on compact manifolds, J. Funct. Anal. 77 (1988) no. 1, p. 123-138.

[13] Suslina T. A., On the absence of eigenvalues of a periodic matrix Schrödinger operator in a layer, Russian Journal of Mathematical Physics 8 (2001), no. 4, p. 463-486.

[14] Thomas L., Time dependent approach to scattering from impurities in a crystal, Commun. Math. Phys. 33 (1973), p. 335-343. 